\title{
MODEL-BASED SOUND SYNTHESIS OF TANBUR, A TURKISH LONG-NECKED LUTE
}

\author{
Cumhur Erkut and Vesa Välimäki \\ Helsinki University of Technology \\ Laboratory of Acoustics and Audio Signal Processing \\ Espoo, Finland \\ Cumhur.Erkut@hut.fi, Vesa.Valimaki@hut.fi
}

\begin{abstract}
Physics-based simulation and sound synthesis of the tanbur, a traditional Turkish long-necked lute is tackled by two computational models based on digital waveguides. A linear generic dual-polarization simulation including sympathetic coupling is calibrated according to recorded sound examples. The other approach utilizes a specific model that incorporates a nonlinear tension modulation mechanism that is pronounced in the tanbur. The nonlinear implementation can accurately reproduce features such as variation of the fundamental frequency, nonlinear coupling of the harmonic partials, and tension modulation driving force coupling to the body. The synthesis results of both models are justified by audio demonstrations available at http://www. acoustics.hut.fi/ cerkut/icassp2000/.
\end{abstract}

\section{INTRODUCTION}

The model-based sound synthesis of the stringed instruments has been an active research field within the last decade. The most efficient synthesis models are based on the theory of digital waveguides [1]. The commuted synthesis $[2,3]$ that is based on the linearity and time-invariance of the synthesis model is an important method for development of a generic string instrument model. Recently, such a model has been presented including consolidated pluck and body wavetables, a pluck-shaping filter, a pluck-position comb filter, string models with loop filters and continuously variable delays, and sympathetic couplings between the strings [4].

The generic linear string-instrument synthesis models are close to their maturity. However, a recent interest in the field is to model a specific string instrument, such as the Finnish kantele [5].

There are two main approaches to simulate a specific string instrument. The first approach uses a generic string synthesis model as a template and tries to achieve realistic synthesis tones by carefully calibrating the model parameters from the recorded sound examples $[6,7,8]$. The second approach is to elaborate the current models by developing new features to simulate unique characteristics of

The authors wish to thank Matti Karjalainen for fruitful discussions. Tero Tolonen's help is highly acknowledged. This work was financed by the Academy of Finland.

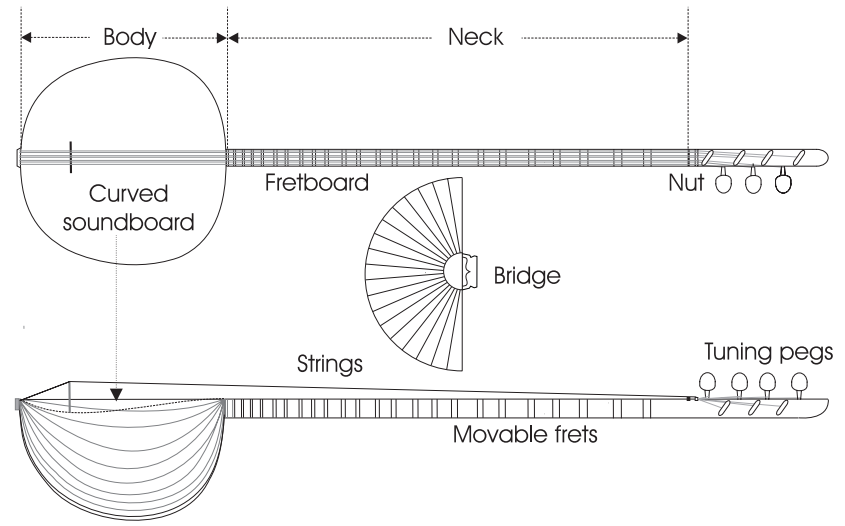

Figure 1: The layout of the tanbur. A small body, a curved soundboard and a very long neck are the main characteristics of the instrument.

the instrument under study. This approach resulted in extended synthesis models in the past [9], often relaxing the assumptions about linearity and time-invariance. Recently, an important family of nonlinear models were unified under the general framework of tension modulation simulation $[10,11]$.

In this study, the model-based sound synthesis of the tanbur is tackled using both generic and specific approaches. The structure of the paper is as follows: In Section 2, we introduce the instrument and summarize its important acoustical properties. In Section 3, we delineate the linear synthesis model for tanbur, and discuss the calibration of the model parameters. Section 4 introduces the nonlinear synthesis model of the tanbur, accounting for the simulation of tension modulation driving force observed in tanbur tones [12]. Finally, in Section 5, we draw our conclusions.

\section{ACOUSTICS OF THE TANBUR}

The tanbur is one of the oldest members of the long-necked lute family [13]. Fig. 1 shows the layout of the tanbur. The instrument has seven strings arranged in three pairs and a single string. A long plectrum is used for plucking, which gives a characteristic scratch during the attack of a tanbur tone. Normally, only one string pair is plucked (we refer to 


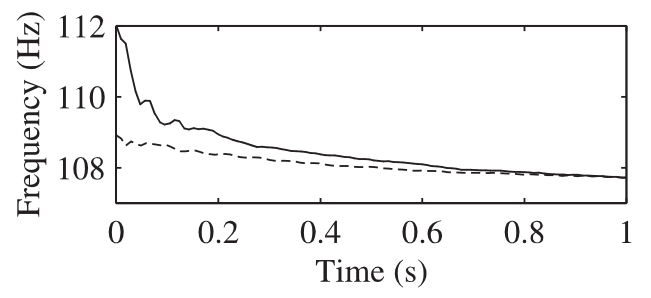

Figure 2: Fundamental frequency drift as detected in tanbur tones plucked moderately (left) and plucked hard (right).

this string pair as the melody pair), the other strings function as resonators. Sometimes, however, the other strings are also plucked to produce pedal tones. The movable nylon frets introduce significant damping during position playing, compared to open strings, but they allow smooth glissandi between the notes. Since the player's right arm is to used to balance the instrument while the right hand plucks the strings, the plucking point is typically constant.

The low-tensioned strings can highly deviate from their rest positions; strong strokes are common in tanbur playing. The violin-like bridge is inserted between the strings and a curved soundboard without gluing, and therefore it has several degrees of freedom.

The vibration of the tanbur strings is relatively nonlinear due to the modulation of the tension. As the following analysis shows, the tension modulation exerts a longitudinal force on the bridge. The construction of the bridge allows this force to be efficiently coupled to the body and radiated as sound. This is quite dissimilar to, e.g., the acoustic guitar where the bridge is glued to the top plate, which results in a relatively rigid structure in the longitudinal direction.

In addition to the longitudinal force output, fundamental frequency variation and coupling of harmonic modes are among the perceptually important effects of tension modulation. The following subsection describes briefly the tension modulation phenomenon and summarizes the acoustical analysis of the tanbur presented in [13].

\subsection{TENSION MODULATION}

Tension modulation depends essentially on the elongation of the string during vibration [14]. The elongation increases the tension of the string which in turn increases the propagation velocity of the transversal waves. This implies that the string vibration is not strictly periodic since the propagation velocity is a function of elongation. Thus, we use the term effective fundamental period to refer to a short-time average value of the period.

Fig. 2 depicts the fundamental frequency variation related to tension modulation detected in a moderately (dashed line) and a hard (solid line) plucked tanbur tone; see [13, Fig. 6] for details. In the hardplucked case, the pitch variation is clearly audible as may be perceived in the audio examples available at http://www.acoustics.hut.fi/ cerkut/icassp2000/. It is clear from the audio examples that the pitch variation caused by tension modulation is important for the character of tanbur tones.
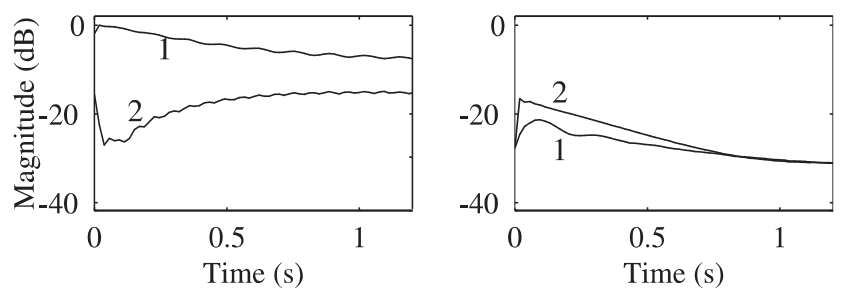

Figure 3: Amplitude envelopes of the first two harmonics of a tanbur tone as detected in string vibration (left), and in vibration of the soundboard (right).

The longitudinal force coupling to the body is investigated in [13]. Particularly, amplitude envelopes of the harmonics of string and body vibration and radiated sound were detected using short-time Fourier analysis. Fig. 3 depicts the envelope of the first and the second harmonic detected in string vibration (left) and in soundboard vibration (right) of a mid-plucked tanbur tone. In the string vibration, the first harmonic is most pronounced, as expected. The second harmonic has a relatively low initial level but the amplitude gradually increases with time. This suggests that the vibration modes are nonlinearly coupled so that energy is transferred back to the string at double the frequency of the first harmonic. In the soundboard vibration, the second harmonic has a higher initial amplitude level than the first one and also a very sharp attack.

Investigating the two figures, it is clear that a linear system cannot produce such a difference in the behavior of the envelopes of the second harmonic. This suggests that the longitudinal force caused by tension modulation produces the second harmonic in the soundboard vibration. For a more detailed discussion including analysis of the radiated sound, see [13]. The experiments of Figs. 2 and 3 demonstrate the importance of tension modulation to the tanbur tones.

\section{THE LINEAR MODEL}

Fig. 4 shows the basic string synthesis model that consists of a feedback loop with three parts: a delay line of $L_{\mathrm{I}}$ unit delays, a fractional delay filter $F(z)$ that implements interpolation between sample values, and a loop filter $H_{l}(z)$ $[15,6]$. The delay line and the fractional delay filter [16] together contribute to the generally real-valued delay of the loop, and thus determine the fundamental frequency of the synthetic tone. A third-order Lagrange interpolation filter is used for the fractional delay filter, and the loop filter $H_{l}(z)$ is a one-pole filter. The main function of the loop filter is to attenuate the waveform of the tone from one period to the next in a natural manner simulating the effect of losses. The transfer function of the string model is $S(z)=1 /\left[1-H_{l}(z) z^{L_{\mathrm{I}}} F(z)\right]$.

The basic model is used to extract fundamental parameters for a tanbur synthesis model from the sound samples recorded in an anechoic room using the calibration scheme described in [7]. For the melody pair, four excitation signals per octave are extracted for three different plucking strengths, along with the loop-filter parameters. For the 


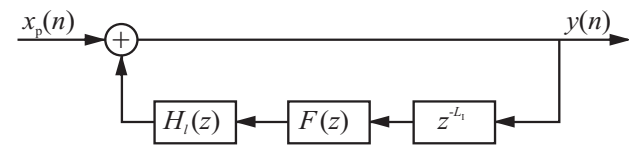

Figure 4: The basic string model $[15,6]$.

resonator strings, only one excitation signal for each of three plucking strengths are extracted to account for the pedal tones. The characteristic plectrum scratches are captured in the excitation signals.

Starting with the basic string synthesis model of Fig. 4, a more detailed model, such as the generic string instrument model [4] is needed for a more realistic simulation of the tanbur. Fig. 5 shows a dual-polarization string model, which we used to model a melody string. A single melody string is modeled with two basic string models $S_{h}(z)$, and $S_{v}(z)$, for the horizontal and vertical polarizations, respectively. The coupling between polarization is controlled with a gain factor $g_{c}$. The remaining resonator strings are modeled using single-polarization models.

The lowpass pluck-shaping filter $E(z)$, which has the same transfer function as the loop-filter, accomplishes two main tasks: It accounts for the damping introduced by nylon frets, and the spectral differences between plucking strengths. $E(z)$ is tuned using the consolidated excitation signals obtained from the basic string model. Note that, as shown in Section 2.1, very strong excitation signals exhibit nonlinear characteristics. Therefore they are not used in the calibration of $E(z)$, and hence the linear model cannot synthesize very strong plucks accurately.

The pluck-position comb filter $P(z)$ is set to a fixed value considering the normal pluck position in tanbur playing, which is approximately $5 \mathrm{~cm}$ away from the bridge.

The remaining parameters of the linear model are manually tuned to give realistic synthesis outputs. An important point to mention is the special structure of the coupling matrix $C$. Unlike the typical case, where every string and fret combination should be analyzed for the extraction of $C$ matrix coefficients, the task is easier for tanbur: The energy is transferred only from the melody pair to the fixed-length resonator strings. However, the extraction of the $C$-matrix coefficients is left as a future task.

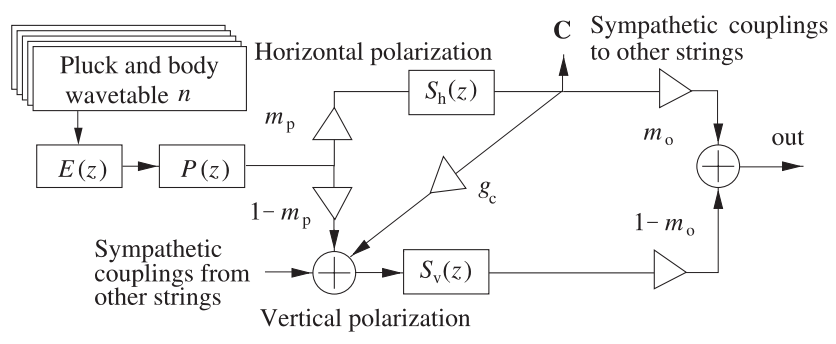

Figure 5: The generic plucked string instrument model [4].

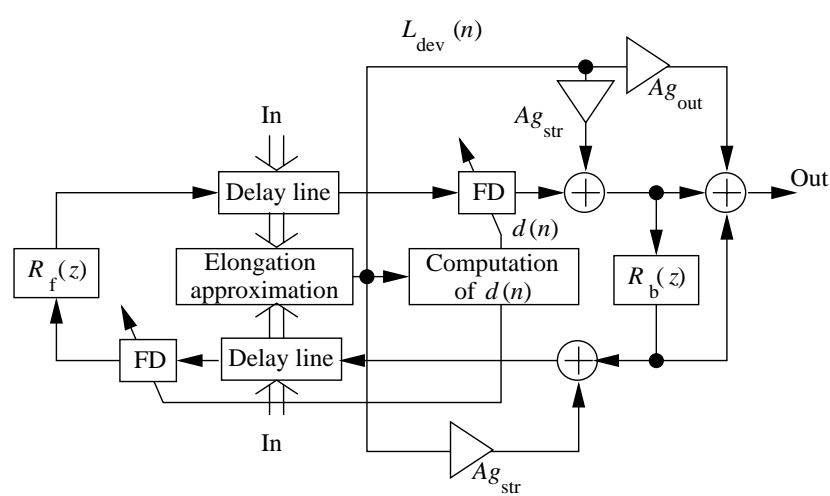

Figure 6: Dual-delay-line waveguide model of a string including tension modulation driving force output and coupling [12].

\section{THE NONLINEAR MODEL}

The block diagram in Fig. 6 depicts a string model that includes the tension modulation in terms of variation of the propagation velocity and of coupling of the longitudinal force to the output and back to the string [12]. The model includes two delay lines with variable delays implemented with fractional delay filters. The wave variables are chosen to be slope waves so that the elongation estimation is straightforward [11]. Reflection filters $R_{\mathrm{b}}(z)$ and $R_{\mathrm{f}}(z)$ model the string terminations. The parameter $g_{\mathrm{str}}$ controls the coupling of the tension modulation back to the string, and parameter $g_{\text {out }}$ determines the longitudinal tension modulation driving force coupling to the body. The $A$ parameter is related to the string properties and nominal tension. Estimation procedures for the tension modulation parameters are described in $[11,12]$. The other model parameters are estimated as in the linear case, cf. Sec. 3.

Fig. 7 shows synthesized tones exhibiting a pitch drift (cf. Fig. 2). The amount of the pitch drift is controlled by varying the amplitude of the initial slope waves. The physical correspondence is clear, the slope waves with larger amplitude values correspond to strongly plucked tanbur tones. In the figure, two examples of moderately and hard plucked tones are shown.

Fig. 8 shows the behavior of the second harmonic for the analyzed and synthesized tanbur tones. The upper plot superimposes the amplitude envelopes of the second harmonics in Fig. 3. Note that the envelopes detected in string vibration (solid) and body vibration (dashed) are arranged for the ease of reading, and their absolute scales are ar-

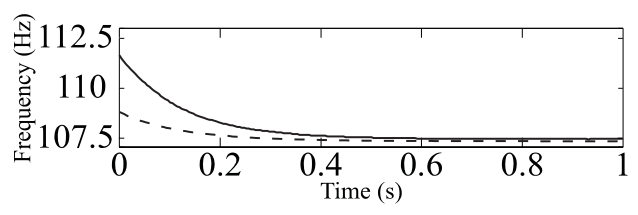

Figure 7: Synthesized tanbur tones to exhibiting pitch drift plucked moderately (left) and plucked hard (right). 


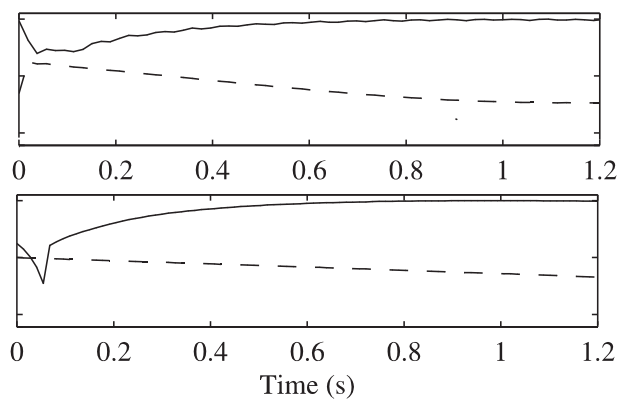

Figure 8: The second harmonic in analyzed (upper plot) and synthesized (lower plot) tones.

bitrary. The lower plot shows the similar behavior in the synthesized tone. The dashed line is the model output corresponding to the physical force at the bridge. To observe the string vibration (solid line) we set the output coupling coefficient $g_{\text {out }}$ to zero, and demonstrate the build-up of the second harmonic. As in the upper plot, the harmonics are scaled for ease of reading. This example shows that the model can simulate the nonlinear phenomena observed in tanbur tones.

A drawback of the tension-modulated model comparing to the linear case is the increase in computation. Depending on the delay-line length, the difference can be one order of magnitude or more. However, even the nonlinear model with a low-pitch tone can easily be implemented in realtime in a typical desktop computer.

\section{CONCLUSIONS}

We have tackled the modeling of the tanbur by a generic linear plucked string instrument model and by a specific nonlinear model that accounts for the tension modulation effects inherently present in tanbur tones. The linear model provides a computationally efficient and tractable synthesis supported by automated parameter analysis. However, the synthetic tones do not include features such as the fundamental frequency variation. The nonlinear model, however, accurately reproduces the desired nonlinear features at the cost of increased computational efforts and a more difficult analysis task. The sound examples available at http://www.acoustics.hut.fi/ cerkut/icassp2000/ demonstrate that realistic tanbur synthesis can be obtained by applying digital waveguides using both linear and nonlinear models.

\section{REFERENCES}

[1] J. O. Smith, "Physical modeling using digital waveguides," Computer Music J., vol. 16, no. 4, pp. 74-91, 1992.

[2] J. O. Smith, "Efficient synthesis of stringed musical instruments," in Proc. Int. Computer Music Conf., (Tokyo, Japan), pp. 64-71, Sept. 1993.

[3] M. Karjalainen, V. Välimäki, and Z. Jánosy, "Towards high-quality sound synthesis of the guitar and string instruments," in Proc. Int. Computer Music Conf., (Tokyo, Japan), pp. 56-63, Sept. 1993.

[4] M. Karjalainen, V. Välimäki, and T. Tolonen, "Plucked-string models: From the Karplus-Strong algorithm to digital waveguides and beyond," Computer Music J., vol. 22, no. 3, pp. 17-32, 1998.

[5] V. Välimäki, M. Karjalainen, T. Tolonen, and C. Erkut, "Nonlinear modeling and synthesis of the kantele-a traditional Finnish string instrument," in Proc. Int. Computer Music Conf., (Beijing, China), pp. 220-223, Oct. 1999.

[6] V. Välimäki, J. Huopaniemi, M. Karjalainen, and Z. Jánosy, "Physical modeling of plucked string instruments with application to real-time sound synthesis," J. Audio Eng. Soc., vol. 44, pp. 331-353, May 1996.

[7] T. Tolonen and V. Välimäki, "Automated parameter extraction for plucked string synthesis," in Proceedings of the Institute of Acoustics, vol. 19, pp. 245-250, Sept. 1997. Presented at the International Symposium on Musical Acoustics, Edinburgh, UK.

[8] V. Välimäki and T. Tolonen, "Development and calibration of a guitar synthesizer," J. Audio Eng. Soc., vol. 46, pp. 766-778, Sept. 1998.

[9] M. Karjalainen, J. Backman, and J. Pölkki, "Analysis, modeling and real-time synthesis of the kantele, a traditional finnish string instrument," in Proc. ICASSP, (Minneapolis, Minnesota), pp. 229-232, IEEE, 1993.

[10] V. Välimäki, T. Tolonen, and M. Karjalainen, "Plucked-string synthesis algorithms with tension modulation nonlinearity," in Proc. ICASSP, vol. 2, (Phoenix, Arizona), pp. 977-980, Mar. 1999.

[11] T. Tolonen, V. Välimäki, and M. Karjalainen, "Modeling of tension modulation nonlinearity in plucked strings," IEEE Trans. Speech and Audio Processing, 1999. Accepted for publication.

[12] T. Tolonen, C. Erkut, V. Välimäki, and M. Krajalainen, "Simulation of plucked strings exhibiting tension modulation driving force," in Proc. Int. Computer Music Conf., (Beijing, China), pp. 5-8, Oct. 1999.

[13] C. Erkut, T. Tolonen, M. Karjalainen, and V. Välimäki, "Acoustical analysis of tanbur, a Turkish long-necked lute," in Proc. Int. Congr. Sound Vibr., vol. 1, pp. 345-352, July 1999.

[14] K. A. Legge and N. H. Fletcher, "Nonlinear generation of missing modes on a vibrating string," J. Acoust. Soc. $A m$. , vol. 76, pp. 5-12, July 1984.

[15] D. A. Jaffe and J. O. Smith, "Extensions of the Karplus-Strong plucked-string algorithm," Computer Music J., vol. 7, no. 2, pp. 56-69, 1983. Also published in Roads C. (ed). 1989. The Music Machine, pp. 481494. The MIT Press. Cambridge, Massachusetts, USA.

[16] T. I. Laakso, V. Välimäki, M. Karjalainen, and U. K. Laine, "Splitting the unit delay-Tools for fractional delay filter design," IEEE Signal Processing Magazine, vol. 13, pp. 30-60, Jan. 1996. 